\title{
On testing substitutability
}

\author{
Cosmina Croitoru ${ }^{\mathrm{a}}$, Kurt Mehlhorn ${ }^{\mathrm{b}}$ \\ ${ }^{a}$ Saarland University, Saarbrücken \\ ${ }^{b}$ Max Planck Institut for Informatics, Saarbrücken
}

\begin{abstract}
The papers [1] and [2] propose algorithms for testing whether the choice function induced by a (strict) preference list of length $N$ over a universe $U$ is substitutable. The running time of these algorithms is $O\left(|U|^{3} \cdot N^{3}\right)$, respectively $O\left(|U|^{2} \cdot N^{3}\right)$. In this note we present an algorithm with running time $O\left(|U|^{2} \cdot N^{2}\right)$. Note that $N$ may be exponential in the size $|U|$ of the universe.
\end{abstract}

Keywords: Choice functions, Substitutability, Algorithm complexity

A choice function on a finite set $U$ of alternatives is any function $f$ from subsets of $U$ to subsets of $U$ that maps any set $A$ to a subset of itself, i.e., $f(A) \subseteq A$ for all $A \subseteq U$. A choice function $f$ is substitutable if

$$
A \subseteq B \text { implies } f(B) \cap A \subseteq f(A) \text { for all } A, B \subseteq U,
$$

i.e. the additional alternatives provided by $B$ do not promote any $x \in A-f(A)$ to the set of selected elements.

We are interested in choice functions induced by preference lists $\mathbb{Y}$ on subsets of $U$. A preference list $\mathbb{Y}$ is simply an ordered list of subsets of $U$ and the associated choice function $f_{\mathbb{Y}}$ maps any subset $A$ of $U$ to the first element on the list that is contained in $A$. If $\mathbb{Y}$ is understood from the context, we write $f$ instead of $f_{\mathbb{Y}}$. We use $N$ to denote the number of elements on $\mathbb{Y}$, and, in order to make $f$ defined for all $A$, we assume that the empty set is the last element of $\mathbb{Y}$. For elements $X$ and $Y$ in $\mathbb{Y}$, we write $X \succ Y$ if $X$ properly precedes $Y$ on $\mathbb{Y}$ and we write $X \succeq Y$ for $X \succ Y$ or $X=Y$.

For example, let $U=\{a, b, c, d\}$ and $\mathbb{Y}=(\{a, b\},\{a, c, d\},\{a, c\},\{a\},\{c\}, \emptyset)$. Then $f_{\mathbb{Y}}(\{a, b, c\})=\{a, b\}$. The function $f_{\mathbb{Y}}$ is not substitutable since $d \in\left(f_{\mathbb{Y}}(\{a, c, d\}) \cap\right.$ $\{d\})-f_{\mathbb{Y}}(\{d\})$. We refer to [1] for a discussion of the role of substitutable choice functions in economics.

$\mathbb{Y}$ is coherent if $X \succ Y$ implies $X \nsubseteq Y$ for any two elements on $\mathbb{Y}$. Assume $X \succ Y$ and $X \subseteq Y$. Then $Y$ does not lie in the range of $f_{\mathbb{Y}}$ and removing $Y$ from $\mathbb{Y}$ does not change the function $f$. Thus we may assume that $\mathbb{Y}$ is coherent.

From now on, $\mathbb{Y}$ denotes a coherent preference list and $f$ stands for $f_{\mathbb{Y}}$. $\mathbb{Y}$ is substitutable if $f$ is a substitutable choice function.

Lemma 1. Let $\mathbb{Y}$ be a coherent preference list on $U$. Then for any $A \subseteq U, f(A)=A$ if and only if $A \in \mathbb{Y}$. 
Proof. Since $f$ maps the powerset of $U$ to $\mathbb{Y}, f(A)=A$ implies $A \in \mathbb{Y}$. Conversely, assume $A \in \mathbb{Y}$ and $f(A) \succ A$. Then $f(A)$ and $A$ are members of $\mathbb{Y}$ with $f(A) \succ A$ and $f(A) \subseteq A$, a contradiction to the coherence of $\mathbb{Y}$.

An established condition of choice functions known as Aizerman's outcast, or Chernoff's postulate $5^{*}$, or $\hat{\alpha}$ (see Brandt and Harrenstein [3]) is

$$
\text { (outcast) : } \quad \text { if } f(A) \subseteq B \subseteq A \text { then } f(B)=f(A) .
$$

Lemma 2. If $Y$ is a coherent preference list on $U$, then $f$ satisfies outcast.

Proof. $B \subseteq A$ implies $f(A) \succeq f(B)$ and $f(A) \subseteq B$ implies $f(B) \succeq f(f(A))=f(A)$, where the last equality uses coherence. Thus $f(A)=f(B)$.

Lemma 3. Let $\mathbb{Y}$ be a coherent and substitutable preference list on $U$. If $X$ is a member of $\mathbb{Y}$ then also every subset of $X$ is a member of $\mathbb{Y}$.

Proof. Assume $X=f(X)$ and $A \subseteq X$. By substitutability, $f(X) \cap A \subseteq f(A)$ and hence $A=X \cap A=f(X) \cap A \subseteq f(A)$. Thus $f(A)=A$.

A preference list $\mathbb{Y}$ is complete if it contains for each $X \in Y$ also all of its subsets. Note that complete preference lists are exponentially long in the size of their largest member.

In order to demonstrate non-substitutability of a preference list, we need to exhibit sets $A$ and $B$ with $A \subseteq B$ and $f(B) \cap(A-f(A)) \neq \emptyset$. We next show that we can restrict the search to special subsets of $U$. A witness (to non-substitutability) is a pair $(X, Y)$ of members of $\mathbb{Y}$ such that $X \succ Y, f(X \cup Y)=X$ and there is an $x \in X-Y$ such that $f(Y \cup\{x\})=Y$. Note that $x$ is selected when the set of alternatives is $X \cup Y$ (this is the set $B$ ) but is not selected when the set of alternatives is $Y \cup\{x\}$ (this is the set $A$ ).

Theorem 4. $\mathbb{Y}$ is not substitutable if and only if there is a witness to non-substitutability.

Proof. Assume first that $(X, Y)$ is a witness. Then $X \succ Y, f(X \cup Y)=X$ and there is an $x \in X-Y$ such that $f(Y \cup x)=Y$. Let $A=Y \cup\{x\}$ and $B=X \cup Y$. Then $A \subseteq B$ and $x \in f(B) \cap(A-f(A))$. Thus $f$ is not substitutable.

Conversely, assume that $f$ is not substitutable. Then there are subsets $A$ and $B$ of $U$ with $A \subseteq B$ and $f(B) \cap A \nsubseteq f(A)$. Since $A \subseteq B$, we have $f(B) \succeq f(A)$. In fact, $f(B) \succ$ $f(A)$ since $f(B)=f(A)$ and $f(A) \subseteq A$ implies $f(B) \cap A=f(A)$. Since $f(A) \subseteq A \subseteq B$, we have $f(A) \cup f(B) \subseteq B$ and hence $f(B) \subseteq f(A) \cup f(B) \subseteq B$. Thus $f(f(A) \cup f(B))=$ $f(B)$ by property (outcast). Let $x \in(f(B) \cap A)-f(A)$. Then $f(B) \cup\{x\} \subseteq A$ and $f(A) \subseteq$ $f(A) \cup\{x\} \subseteq A$ and hence $f(f(A) \cup\{x\})=f(A)$ by (outcast). Thus $(f(B), f(A))$ is a witness.

Theorem[4 directly translates into an algorithm of running time $O\left(N^{3}|U|+N^{2}|U|^{2}\right)$. Note first that one can determine $f(A)$ in time $O(N|U|)$ by simply scanning the list $\mathbb{Y}$ and checking each set for containment. The algorithm has two phases. In the first phase, one determines for each $Y \in \mathbb{Y}$ the set of $x$ for which $f(Y \cup\{x\})=Y$. This requires $N|U|$ function evaluations and $O\left(N^{2}|U|^{2}\right)$ time. Then one checks for every pair $(X, Y)$ of elements of $\mathbb{Y}$, whether it is a witness. This requires $N^{2}$ function evaluations and $N^{2}|U|$ look-ups of precomputed values and hence takes time $O\left(N^{3}|U|\right)$. 


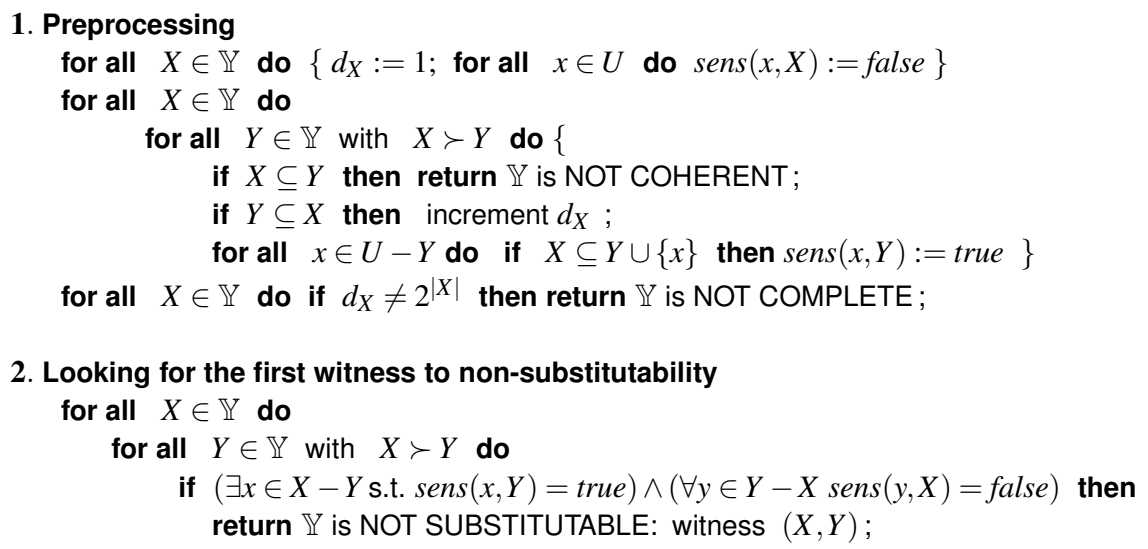

Figure 1: Testing if the list $\mathbb{Y}$ is substitutable

We improve the running time to $O\left(N^{2}|U|^{2}\right)$. The crucial insight is as follows. We search for a witness pair $(X, Y)$ in increasing order of $X$. Of course, we stop the search as soon as we have found a witness. So when we consider a pair $(X, Y)$ we know that there is no witness $(Z, \cdot)$ with $Z \succ X$. We then have $f(X \cup Y)=X$ if and only if $f(X \cup\{x\})=X$ for all elements $x \in Y-X$. We stress that this equivalence does not hold in general, it only holds under the assumption that there is no earlier witness. So we can replace the function evaluation $f(X \cup Y)$ of cost $O(N|U|)$ by $|U|$ look-ups of precomputed values. We next give the details.

We call $X \in \mathbb{Y}$ insensitive to $x \in U$ if $f(X \cup\{x\})=X$ and sensitive otherwise.

Lemma 5. Let $X, Y \in \mathbb{Y}$ with $X \succ Y$. If $f(X \cup Y)=X$, then $X$ is insensitive to all $x \in Y-X$. If $X$ is insensitive to all $x \in Y-X$ and there is no witness $(Z, \cdot)$ with $Z \succ X$, then $f(X \cup Y)=X$.

Proof. Let $x \in Y-X$ be arbitrary. Then $X \subseteq X \cup\{x\} \subseteq X \cup Y$ and hence $X=f(X \cup Y) \succeq$ $f(X \cup\{x\}) \succeq f(X)=X$. Thus $f(X \cup\{x\})=X$ and $X$ is insensitive to $x$.

For the second part, assume $f(X \cup Y)=Z$ with $Z \succ X$. Then $Z \subseteq X \cup Y$ and hence $Z \cup X \subseteq X \cup Y$. Thus $Z \succeq f(X \cup Y) \succeq f(X \cup Z) \succeq Z$, where the last inequality follows from $Z \subseteq X \cup Z$. Thus $f(X \cup Z)=Z$. Since $(Z, X)$ is not a witness, we must have $f(X \cup\{x\}) \neq X$ for every $x \in Z-X$. On the other hand, $Z-X \subseteq Y-X$ (since $Z \subseteq X \cup Y$ ) and $f(X \cup\{x\})=X$ since $X$ is insensitive to all $x \in Y-X$, a contradiction.

Lemma 5 suggests a way to find the non-substitutability witness $(X, \cdot)$ with minimal first component. 
Theorem 6. Let $X, Y \in \mathbb{Y}$ with $X \succ Y$ and assume that there is no witness $(Z, \cdot)$ with $Z \succ X$. Then $(X, Y)$ is a witness if and only if $X$ is insensitive to all $x \in Y-X$ and $Y$ is sensitive to some $x \in X-Y$.

Proof. Assume first that $(X, Y)$ is a witness pair. Then $Y$ is sensitive to some $x \in X-Y$ and $f(X \cup Y)=X$. The latter implies that $X$ is insensitive to all elements of $Y-X$.

Conversely, assume that $X$ is insensitive to all $x \in Y-X$ and $Y$ is sensitive to some $x \in X-Y$. Then, $f(X \cup Y)=X$ by Lemma 5 and hence $(X, Y)$ is a witness pair.

We are now ready for the algorithm. The algorithm has two phases. In a preprocessing phase, we determine whether $\mathbb{Y}$ is coherent, complete, and, most importantly, compute the Boolean flags sens $(x, X)$ which is true if $X \in \mathbb{Y}$ is sensitive to $x$.

In the main computation, we search for the first witness to non-substitutability. We iterate over the elements of $X$ of $\mathbb{Y}$ in increasing order. Assume that there is no witness $(Z, \cdot)$ with $Z \succ X$. We then iterate over the $Y \in \mathbb{Y}$ with $X \succ Y$ and use Theorem 6 to determine whether $(X, Y)$ is a witness pair.

The most expensive task of the first phase is the construction of the Boolean matrix sens of size $|U| \times N$. Since an inclusion test needs $O(|U|)$ time, the overall time is therefore $O\left(|U|^{2} \cdot N^{2}\right)$. The time complexity of the second phase is $O\left(|U| \cdot N^{2}\right)$ (the $|U|$ factor is given by the inspection of the Boolean matrix sens in order to apply Theorem 6).

By Theorems 4 and 6 and the above discussion, the following corollary holds.

Corollary 1. The algorithm in Figure \tests in $O\left(|U|^{2} \cdot N^{2}\right)$ time if a given preference list of size $N$ over an universe $U$ is substitutable.

Remarks. The $O(N)$ speed-up over the existing algorithms is significant since (as we noted after the definition of complete lists) $N$ is exponential in the size of the largest member of $\mathbb{Y}$. The algorithm in [2] also applies to weak preferences. We leave it as an open problem whether this also holds for our algorithm.

\section{References}

[1] J. W. Hatfield, N. Immorlica, S. D. Kominers, Testing substitutability, Games and Economic Behavior (2011) 639-645.

[2] H. Aziz, M. Brill, P. Harrenstein, Testing substitutability of weak preferences, Mathematical Social Sciences (2013) 91-94.

[3] F. Brandt, P. Harrenstein, Set-retionalizable choice functions and self- stability, Journal of Economic Theory 146 (2011) 233-273. 